\title{
MORTALIDAD Y SALUD
}

\author{
Dr. HUGO E. BARRERA A., \\ Departamento del Seguro Social, Quito.
}

Para el estudio de algunas variables demcgráficas, en el Ecuador y en casi todos los países de América y del mundo, se disponen de estadísticas veraces y completas. Para el efecto, la División de Estadística y Censos de la Junta Nacional de Planificación, publica anualmente los anuarios estadísticos y su última publicación en esta materia corresponde al Anuario de Estadísticas Vitales de 1965 y, pronto, entrará en prensa el de 1966, del cual he tcmado los principales datos.

Sólo nos ocuparemos de dos procesos de la mortalidad: la mortalidad general y la mortalidad infan. til.

\section{1. a) MORTALIDAD GENERAL. -}

Se conoce con esta denominación, al total de defunciones por todas las causas que ccurren en una colectividad en un período de tiempo que generalmente es un año. Para medir la intensidad del fenómeno, se disponen de ciertos indicadores que se denominan tasas o coeficientes y en el caso que nos ocupa tenemos la tasa cruda o bruta de mortalidad o simplemente "tasa de mortalidad general", y se la expresa diciendo que es el número de individuos que fallecen, por tcdas las causas, en un año, por cada 1.000 habitantes, que en fórmula se la expresa así:

$N$ : totol de defunciones en 1 año MG $=\frac{\text { Población }}{\text { P }} \times 1.000$

En 1966, en el Ecuador han fallecido 59.618 individuos, y como el país, para mediados del mismo año, tuvo una población de 5’325.900 habitantes, por lo mismo si en la fórmula anterior reemplazamos valores, tenemos:

$$
\frac{59.618}{5^{\prime} 325.900} \times 1.000
$$

que nos da una tasa igual a 11,2 por cada 1.000 habitantes. Sólo en esta forma podemos conocer la intensidad - fuerza de ese hecho demográfico llamado mortalidad, comparando con años anteriores en el mismo país o con otros países similares al nuestro y, a veces, para ver el contraste marcado con los Estados Unidos de Norte América.

\section{1. b) LA MORTALIDAD GENERAL EN EL ECUADOR. - En la ta-}

bla I se inserta una serie cronológica, en quinquenios, de tasas de mortalidad general en el Ecuador desde 1920 a 1965. 
TABLA

TASAS DE MORTALIDAD GENERAL EN EL ECUADOR DESDE 1920 A 1965

\begin{tabular}{cc} 
Años & $\begin{array}{c}\text { Tasas por } \\
1.000\end{array}$ \\
\hline 1920 & 28,9 \\
1925 & 27,6 \\
1930 & 24,9 \\
1935 & 24,6 \\
1940 & 20,9 \\
1945 & 18,9 \\
1950 & 16,3 \\
1955 & 14,7 \\
1960 & 14,7 \\
1965 & 11,8 \\
\hline
\end{tabular}

Se cbserva en esta tabla que en 45 años, la mortalidad en el Ecuador ha sufrido un marcado descenso que se traduce por un $59.2 \%$ de reducción, - lo que es lo mismo, hay $1,3 \%$ de reducción anual.

$\mathrm{Si}$ se representarian gráficamente las curvas de natalidad y mortalidad en el Ecuador desde 1945 a 1966, podría apreciarse que la tendencia de la primera tiene una muy ligera disminución, pudiéndose casi afirmar que la línea trazada sobre los puntos, prácticamente es horizontal; en cambio, en la segunda, la tendencia es al descenso, pues la línea trazada sobre los puntos es prácticamente inclinada hacia abajo, separándose cada vez más

TABLA $\|$

MORTALIDAD GENERAL EN ALGUNOS PAISES DE AMERICA: 1925 - 1965 POR DECENIO. TASAS REDONDEADAS POR 1,000 HABITANTES

\begin{tabular}{|c|c|c|c|c|c|c|c|}
\hline \multirow[t]{2}{*}{$P A \mid S E S$} & \multicolumn{5}{|c|}{ AÑOS POR DECENIO } & \multirow{2}{*}{$\begin{array}{c}\% \\
\text { DE REDUC. } \\
\text { en } 40 \text { años }\end{array}$} & \multirow{2}{*}{$\begin{array}{c}\% \\
\text { REDUC. } \\
\text { ANUAL }\end{array}$} \\
\hline & 1925 & 1935 & 1945 & 1955 & 1965 & & \\
\hline ARGENTINA & 13 & 11 & 10 & 9 & 8 & $38 \%$ & 0,9 \\
\hline COSTA RICA & 23 & 20 & 14 & 10 & 8 & $65 \%$ & 1,6 \\
\hline CHILE $\quad 000$ & 25 & 23 & 17 & 12 & 11 & $56 \%$ & 1,4 \\
\hline COLOMBIA & 15 & 16 & 15 & 12 & 12 & $20 \%$ & 0,5 \\
\hline ECUADOR & 28 & 25 & 19 & 15 & 12 & $57 \%$ & 1,4 \\
\hline GUATEMALA & 30 & 27 & 24 & 20 & 17 & $43 \%$ & 1,1 \\
\hline HONDURAS & 16 & 16 & 14 & 10 & 9 & $43 \%$ & 1,1 \\
\hline MEXICO & 25 & 23 & 18 & 12 & 9 & $63 \%$ & 1,5 \\
\hline PERU & 13 & 15 & 12 & 11 & 8 & $38 \%$ & 0,9 \\
\hline URUGUAY & 12 & 11 & 9 & 9 & 9 & $25 \%$ & 0,6 \\
\hline VENEZIJELA & 19 & 18 & 13 & 9 & 9 & $52 \%$ & 1,3 \\
\hline ESTADOS UNIDOS & 12 & 11 & 10 & 9 & 9 & $25 \%$ & 0,6 \\
\hline
\end{tabular}


de la curva de la natalidad, quedando entre las dos, la franja que corresponde al crecimiento vegetativo que, progresivamente, va aumentando hasta alcanzar tasas de 32,1 por 1.000 habitantes, o lo que es lo mismo un $3.2 \%$ de crecimiento vegetativo.

\section{1. c) MORTALIDAD EN AMERICA.}

- Esta tendencia al descenso de la mortalidad general, se repite en todos los países de América y del mundo, en mayor o menor intensidad, como apreciaremos en la tabla $\mathrm{H}_{\text {, }}$ en la cual hemos tomado unos pocos países similares al nuestro y otros muy distintos como Argentina y más aún como los Estados Unidos.

En esta tabla hemos anotado las tasas de mortalidad desde 1924 a 1965 en decenios, para facilitar rápidamente su comprensión y permitir la comparación del fenómeno. También se tha calculado el porcentaje de reducción en 40 años y el tanto por ciento anual en las dos últimas co. lumnas, apreciándose una cierta igualdad en casi todos los países, a excepción de dos o tres; pero la tasa actual de mortalidad en el Ecuador es igua! a la que Uruguay y Estados Unidos tenian en 1925.

1. id) CAUSAS DE DEFUNCION.Tanto los certificados de defunción extendidos por los médicos, como aquellos extendidos por autoridades civiles, parroquiales o cantonales, se someten a un prolijo examen

TABLA II

\section{DIEZ PRINCIPALES GRUPOS DE CAUSAS DE MUERTE Y PORCENTAJE DE CERTIFICACION MEDICA EN EL ECUADOR.-1966}

\begin{tabular}{|c|c|c|c|c|c|}
\hline 8400.80000000 & & $\begin{array}{l}\text { DEFUNC } \\
\text { TOTA }\end{array}$ & $\begin{array}{l}\text { CNESES } \\
\text { LES }\end{array}$ & $\begin{array}{l}\text { DEFUNCIOI } \\
\text { CERTIFIC. }\end{array}$ & $\begin{array}{l}\text { SCON } \\
\text { EDICO }\end{array}$ \\
\hline & & No DEF. & $\%$ Total & No & $\%$ \\
\hline TOTAL REPUBLICA & & 59.618 & 100,0 & 23.561 & 39,5 \\
\hline ENF. APAR. RESPIRATORIO & 10 & 10.374 & 17,4 & 3.367 & $-\overline{32,5}$ \\
\hline SINT. Y EST. MAL DEFIN. & $2^{\circ}$ & 10.216 & 17,1 & 1.287 & 12,6 \\
\hline ENF. INFECC. Y PARASIT. & $3^{\circ}$ & 9.231 & 15,5 & 3.052 & 33,1 \\
\hline ENF. APARATO DIGESTIVO & $4^{\circ}$ & 8.585 & 14,4 & 5.059 & 58,9 \\
\hline ENF. PROP. $1^{\text {a }}$ INFANCIA & $5^{\circ}$ & 4.681 & 7,8 & 1.857 & 39,7 \\
\hline SENILIDAD & $6^{\circ}$ & 3.320 & 5,6 & 467 & 15,0 \\
\hline ACCID. ENV. Y VIOLENC. & $7^{\circ}$ & 2.930 & 4,9 & 1.811 & 611,8 \\
\hline ENF. APARATO CIRCULAT. & $8^{\circ}$ & 2.258 & 3,8 & 1.507 & 66,7 \\
\hline TUMORES & $9^{\circ}$ & 1.940 & 3,2 & 1.773 & 91,4 \\
\hline OTRAS CON MEN. $3 \% \mathrm{c} / \mathrm{u}$. & $10^{\circ}$ & 6.083 & 10,2 & 3.351 & 55,0 \\
\hline
\end{tabular}


con un personal entrenado en la clasificación de enfermedades y causas de defunción, en la imisma Junta $\mathrm{Na}$ cional de Planificación. De esta manera las tabulaciones ordenadas de acuerdo al mayor número de defunciones, nos permiten presentar la tabla lll, en la cual se observa que las enfermedades del aparato respiratorio, ocupan el primer puesto como principal causa de defunción en el Ecuador en 1966, con un 17,4\%; siguiendo luego, el orden de frecuencia, tenemos el denominado "síntomas de estado mal definido" con un $17.1 \% ; y$, ocupando el tercer puesto, el grupo de enfermedades infecciosas y parasitarias que representan el $15,5 \%$.

\section{1. e) LAS ENFERMEDADES INFEC- CIOSAS Y PARASITARIAS}

En nuestro pais, este rubro es muy importante, cuando en otros países casi ya no se las conoce o se registran en número tan bajo que ocupan el $8^{\circ}, 9^{\circ}$ - $10^{\circ}$ puesto como causa de defunción. Tabla IV, En esta tabla igual que en la anterior se han colocado las causas de muerte según la frecuencia

TABLA IV

DOCE PRINCIPALES GRUPOS DE ENFERMEDADES INFECCIOSAS Y PARASITARIAS Y PORCENTAJES DE CERTIFICACION MEDICA. ECUADOR: 1966

\begin{tabular}{|c|c|c|c|c|c|}
\hline 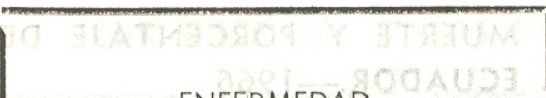 & $\overline{902}$ & $\begin{array}{l}\text { DEFUN } \\
\text { TOTA }\end{array}$ & $\begin{array}{l}\text { NIES } \\
\text { ES }\end{array}$ & $\begin{array}{l}\text { DEFUNICIOI } \\
\text { CERTIFIC. }\end{array}$ & $\begin{array}{l}\text { ES CON } \\
\text { MEDICO }\end{array}$ \\
\hline ENFERMEDI & & NN DEF. & $\%$ Total & No & $\%$ \\
\hline TOTAL REPUBLICA & & 9.231 & 100,0 & 3.052 & 33,0 \\
\hline TOSFERINA & 10 & 3.044 & 33,0 & 255 & 8,4 \\
\hline TETANOS & $2^{\circ}$ & 1.635 & 17,7 & 716 & 43,8 \\
\hline SARAMPION & $3^{\circ}$ & 1.500 & 16,2 & 322 & 21,5 \\
\hline TB., TODAS LAS FORMAS & $4^{\circ}$ & 1.302 & 14,1 & 1.019 & 78,3 \\
\hline HELMINTIASIS & $5^{\circ}$ & 221 & 2,3 & 132 & 59,7 \\
\hline DISENTERIAS TODAS FORM. & $6^{\circ}$ & 216 & 2,3 & 46 & 21,3 \\
\hline PALUDISMO & $7^{\circ}$ & 176 & 1,9 & 23 & 13,0 \\
\hline TIFOIDEA Y SALMONELLA & $8^{\circ}$ & 161 & 1,7 & 116 & 72,0 \\
\hline SEPTICEMIA Y PIEMIA & $9^{\circ}$ & 93 & 1,0 & 87 & 93,5 \\
\hline POLICMIELITIS Y ENCEF. & $10^{\circ}$ & 72 & 0,8 & 59 & 81,9 \\
\hline DIFTERIA & 119 & 43 & 0,5 & 37 & 86,0 \\
\hline TODAS LAS DEM. INFEC. PAR. & $12^{\circ}$ & 768 & 8,5 & 240 & 31,2 \\
\hline
\end{tabular}


y el tanto por ciento de certificación médica; observándose que la tosferina ha significado más de 3.000 defunciones y por lo tanto ocupa el primer puesto, siguiéndolla el tétanos, sarampión y tuberculosis, que en total son responsables del $80 \%$ de las infecciosas y parasitarias. Recuérdese que muchas de estas enfermedades que han sido causa de la muerte de 9.231 personas, pudieron haberse evitado con la vacunación o un buen tratamiento médico, ya sea de consulta externa o de internación en medios hospitalarios.

\section{2. a) LA MORTALIDAD INFAN-}

TIL.-Es el thecho demográfico más importante en salud públlica, $y$ es uno de los mejores indicadores que miden el estado sanitario de un país. Para poder medir y por tanto describir y comparar la mortalidad infantil, existe el índice denominado "tasa de mortalidad infantil", y que corresponde al número de defunciones de niños menores de un año por cada 1.000 nacimientos vivos en el lapso de un añó calendario. Por lo tanto, su fórmula es la siguiente:

\section{NNo de defunciones de ni- ños menores de un año \\ T. de M.I. $=\frac{}{\text { No de nacimientos vivos }} \times 1.000$}

Para el Ecuador, en 1966 se registraron 19.977 defunciones de menores de un año y 220.930 nacimientos vivos. Reemplazando valores obtenemos una tasa de mortalidad infantil igual a 90,4 por 1.000 nacidos vivos en 1966.

Esta tasa debe manejarse con mucho cuidado, pues fallas en el numerador $o$ en el denominador pueden producir grandes variaciones que darán una idea falsa del verdadero problema del país. A este respecto, es bien conocido el hecho de la existencia de un sub-registro de defunciones que alcanza, según estimación de la Junta Nacional de Planificación, hasta el $25 \%$ en algunas provincias del país, es decir que no todas las defunciones se inscriben sino que se procede a la sepultación sin inscripción previa en el Registro Civil. A más de esto, el numerador puede estar también afectado por el redondeo de la edad, ya que fallecidos de 11 meses - menos, se les dice de un año de edad, por lo cual no se los toma en cuenta en este tipo de cálculo, yendo a formar parte de la mortalidad de 1 a 2 años o en el grupo de 1 a 4, más frecuentemente. A este respecto, en el país no se ha llegado a la identificación de las partidas de nacimiento y defunción, para determinar con exactitud la edad del fallecido, problema bastante difícil y que en otros países ya se ha solucionado a nivel del Registro Civil. Asimismo el denominador no siempre es completo, pues no todos los nacimientos vivos se inscriben y su inscripción la dejan para otra época, produciéndose el hecho de las inscripcicnes tardías, que disminuyen el denominador.

En la tabla $V$ se presentan las tasas de mortalidad infantil, por decenios, en algunos países de América y el porcentaje de reducción.

Se aprecia que en el Ecuador la tasa ha descendido en un $45 \%$ en 40 años pero al comparar con otros países, en 1965, el Ecuador está en segundo lugar, después de Chile. Pero en este país, las estadísticas son muy biein llevadas y se procede a verificar el nacimiento con la defunción. para determinar la edad del fallecido. Ahora bien, si miramos la tasa de mortalidad infantil de 1925 en Estados Unidos y comparamos con Ecuador, diremos que está atrasado por lo menos cincuenta años en relación al gran país del norte.

Es muy interesante observar la tabla VI, que representa los porcentajes del total, por grupos de edad, de 
TABLA V

TASAS DE MORTALIDAD INFANTIL Y PORCIENTO DE REDUCCION, POR DECENIOS, EN ALGUNOS PAISES AMERICANOS

\begin{tabular}{|c|c|c|c|c|c|c|c|}
\hline PAISES & 1925 & $\begin{array}{l}\text { AÑC } \\
1935\end{array}$ & $\begin{array}{l}\text { POR DE } \\
1945\end{array}$ & $\begin{array}{l}\text { ENIOS } \\
1955\end{array}$ & 1965 & $\begin{array}{l}\% \text { reduc. } \\
\text { en } 40 \text { años }\end{array}$ & $\begin{array}{l}\% \text { redu } \\
\text { anual }\end{array}$ \\
\hline Argentina & 111 & 98 & 74 & 62 & 61 & $45 \%$ & 1,1 \\
\hline Costa Rica & 169 & 144 & 100 & 76 & 75 & $56 \%$ & 1,4 \\
\hline Chile & 224 & 212 & 150 & 117 & 107 & $52 \%$ & 1,3 \\
\hline Colombia & 133 & 116 & 142 & 101 & 82 & $38 \%$ & 0,9 \\
\hline ECUADOR & 170 & 149 & 127 & 107 & 93 & $45 \%$ & 1,1 \\
\hline Guatemala & 94 & 104 & 109 & 97 & 94 & $0 \%$ & 0 \\
\hline Honduras & 101 & 99 & 92 & 57 & 41 & $59 \%$ & 1,5 \\
\hline México & 194 & 127 & 104 & 78 & 61 & $68 \%$ & 1,7 \\
\hline Perú & $\ldots$ & 113 & 109 & 99 & 91 & $19 \%$ & 0,6 \\
\hline Uruguay & 101 & 94 & 57 & 50 & $\ldots$ & $50 \%$ & 1,6 \\
\hline Venezuela & 150 & 135 & 97 & 64 & 48 & $67 \%$ & 1,7 \\
\hline Estados Unidos & 69 & 53 & 33 & 26 & 25 & $64 \%$ & 1,6 \\
\hline
\end{tabular}

TABLA VI

PORCIENTO DE LA MORTALIDAD GENERAL EN 3 DIFERENTES GRUPOS DE EDADES, EN ALGUNOS PAISES DE AMERICA. 1965

P A ISES

TOTAL GRUPOS DE EDAD

menos de 10 años

\begin{tabular}{|c|c|c|c|c|c|c|}
\hline . . . . & . . . . & 100,0 & 33,4 & 7,6 & 1,5 & $42,5 \%$ \\
\hline Colombia & . & 100,0 & 30,7 & 17,2 & 3,5 & $51,4 \%$ \\
\hline ECUADOR & . & 100,0 & 35,0 & 22,3 & 3,5 & $60,8 \%$ \\
\hline México . . . . & . & 100,0 & 28,3 & 14,7 & 3,4 & $46,4 \%$ \\
\hline Perú (1963) & . & 100,0 & 30,8 & 19,9 & 3,2 & $53,9 \%$ \\
\hline Venezuela & . & 100,0 & 29,2 & 11,9 & 3,0 & $44,1 \%$ \\
\hline Estados Unidos. & . & 100,0 & 4,0 & 0,8 & 0,5 & $5,3 \%$ \\
\hline
\end{tabular}


la mortalidad general en algunos países de América en 1965.

Del total de defunciones, el 30\% se produce en los menores de un año, y para el Ecuador ell porcentaje sube al $35 \%$, el más alto de América, pudiendo decir lo mismo al referirse al grupo de 1 a 4 años, en los cuales el total de fallecimientos representa el $22,3 \%$. Para los menores de 10 años, estos porcentajes están sobre el $40 \%$ en algunos países, pero en el Ecuador llega casi al $61 \%$, lo que podría traducirse por el poco "chance" que tiene el ecuatoriano que nace de llegar a viejo. En Estados Unidos, puede cbservarse el bajísimo porcentaje de defunciones que existen en las edades infantiles, y el "chance" de morir de viejo es la regla general.

Para terminar, veamos la tabla VII, que se refiere a las causas de de- función en los menores de un año, ordenadas de acuerdo a su frecuencia y que se expresan en números absolutos y relativos, esto es, con el porcentaje del total:

En esta tabla que totaliza a todas las defunciones ocurridas en los menores de un año, se puede apreciar que la principal causa de muerte es aquella que se agrupa con la denominación de "enfermedades propias de la primera infancia" siendo éstas las respcnsables de los decesos en los niños menores de un mes exactamente, y que en Salud Pública se conoce con el nombre de mortalidad neonatal o mortalidad infantil temprana o del primer mes de vida, edad que es la más vulnerabile dentro del primer año de vida. Las otras enfermedades que vienen a continuación en la tabla VII son en verdad evitables, como to

\section{PRINCIPALES CAUSAS DE MORTALIDAD INFANTIL EN EL ECUADOR EN LOS MENORES DE UN AÑO}

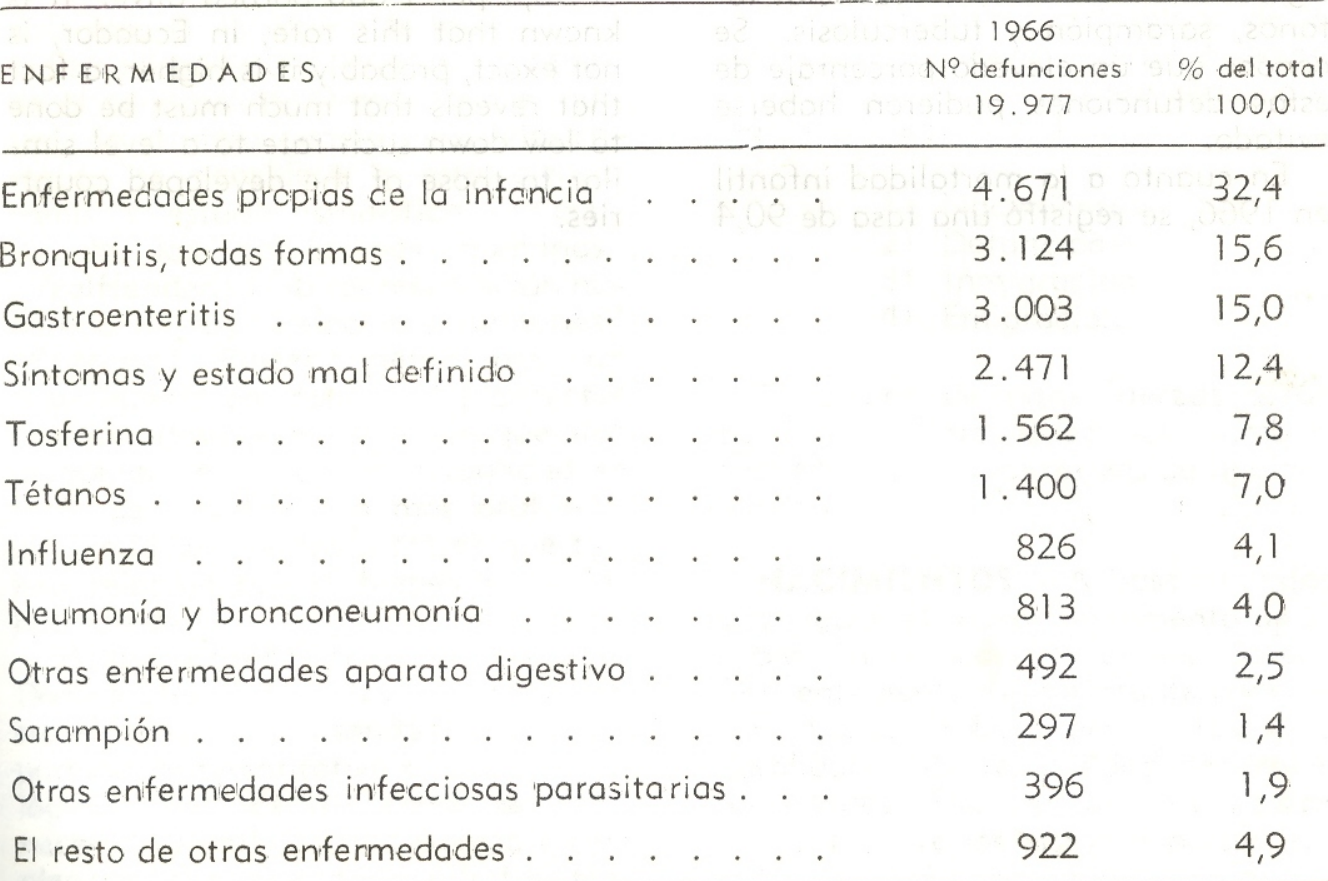


TABLA $V$

TASAS DE MORTALIDAD INFANTIL Y PORCIENTO DE REDUCCION, POR DECENIOS, EN ALGUNOS PAISES AMERICANOS

\begin{tabular}{|c|c|c|c|c|c|c|c|}
\hline PAISES & 1925 & $\begin{array}{l}\text { AÑ } \\
1935\end{array}$ & $\begin{array}{l}\text { POR DE } \\
1945\end{array}$ & $\begin{array}{l}\text { NiOS } \\
1955\end{array}$ & 1965 & $\begin{array}{l}\% \text { reduc. } \\
\text { en } 40 \text { años }\end{array}$ & $\begin{array}{l}\% \text { reduc. } \\
\text { anual }\end{array}$ \\
\hline Argentina & 111 & 98 & 74 & 62 & 61 & $45 \%$ & 1,1 \\
\hline Costa Rica & 169 & 144 & 100 & $=76$ & 75 & $56 \%$ & 1,4 \\
\hline Chile & 224 & 212 & 150 & 117 & 107 & $52 \%$ & 1,3 \\
\hline Colombia & 133 & 116 & 142 & 101 & 82 & $38 \%$ & 0,9 \\
\hline ECUADOR & 170 & 149 & 127 & 107 & 93 & $45 \%$ & 1,1 \\
\hline Guatemala & 94 & 104 & 109 & 97 & 94 & $0 \%$ & 0 \\
\hline Honduras & 101 & 99 & 92 & 57 & 41 & $59 \%$ & 1,5 \\
\hline México & 194 & 127 & 104 & 78 & 61 & $68 \%$ & 1,7 \\
\hline Perú & $\ldots$ & 113 & 109 & 99 & 91 & $19 \%$ & 0,6 \\
\hline Uruguay & 101 & 94 & 57 & 50 & . & $50 \%$ & 1,6 \\
\hline Venezuela & 150 & 135 & 97 & 64 & 48 & $67 \%$ & 1,7 \\
\hline Estados Unidos & 69 & 53 & 33 & 26 & 25 & $64 \%$ & 1,6 \\
\hline
\end{tabular}

TABLA VI

PORCIENTO DE LA MORTALIDAD GENERAL EN 3 DIFERENTES GRUPOS DE EDADES, EN ALGUNOS PAISES DE AMERICA. 1965

PAISES

GRUPOSDEEDAD \\% de defunción TOTAL - 1 año 1 a 4 in

menos de 10 años

\begin{tabular}{|c|c|c|c|c|c|c|c|c|c|c|}
\hline Chile. & . & . & . & . & . & $\quad 100,0$ & 33,4 & 7,6 & 1,5 & $42,5 \%$ \\
\hline Colombia. & . & . & . & . & . & 100,0 & 30,7 & 17,2 & 3,5 & $51,4 \%$ \\
\hline ECUADOR & . & . & . & . & . & 100,0 & 35,0 & 22,3 & 3,5 & $60,8 \%$ \\
\hline México . . & . & . & . & . & . & 100,0 & 28,3 & 14,7 & 3,4 & $46,4 \%$ \\
\hline Perú (1963) & & . & . & . & . & 100,0 & 30,8 & 19,9 & 3,2 & $53,9 \%$ \\
\hline Venezuela & . & . & . & . & . & 100,0 & 29,2 & 11,9 & 3,0 & $44,1 \%$ \\
\hline Estados Unido & & . & . & . & . & 100,0 & 4,0 & 0,8 & 0,5 & $5,3 \%$ \\
\hline
\end{tabular}

